\title{
Fungal Bio Degradation of Carbofuran Pesticide
}

\author{
Kanne Yamene Devi, Priya Iyer \\ PG and Research Department of Biotechnology, Women`s Christian College, Chennai
}

\begin{abstract}
Soil samples collected from various parts in Tamil Nadu for Carbofuran degradation. Soil samples were cultured with PDA and SDA to get fungal isolate. Effect of concentration of pesticide on fungi growth was analyzed. Thin layer chromatography was carried out to confirm degradation of carbofuran. Using HPLC technique carbofuran degradation was analyzed for higher degradation showed fungi.
\end{abstract}

Keywords: SDA, PDA, LPCB staining, carbofuran, TLC, HPLC

\section{INTRODUCTION}

The present study was conducted with an aim to isolate local strains of insecticide degrading fungi and evaluates their characteristics for purposes of bioremediation of insecticide contaminated soil in general, but carbofuran in particular, contaminating fields. These isolates will have the potential to clean-up the environment from such persistent pollutants. In future these fungal strains may be conveniently used in a microbe based system. The present study will provide a baseline data for future studies in biodegradation and bioremediation of carbofuran insecticide.

The indiscriminate and unplanned use of agrochemicals have caused serious environmental problem. The residues of these agrochemicals directly or indirectly gain entry into the food chain and prove health hazardous to animal and plants. The pesticide or insecticide does not readily disappear from the environment, soil microorganism may be responsible for the removal of insecticide. Several factors are involved in insecticide degradation like transformation, photochemical mechanism, physical mechanism, chemical mechanism, microbial degradation and bioremediation. Bioremediation can be defined as any process that uses microorganisms, fungi, green plants or their enzymes to return the natural environment altered by contaminants to its original condition. Bioremediation may be employyed to attack specific soil contaminants. Clean up Biotransformation of various pollutants is a sustainable way to contaminated environments. These bioremediation and biotransformation methods harness the naturally occurring, microbial catabolic diversity to degrade, transform or accumulate a huge range of compounds including hydrocarbons, polychlorinated polyaromatic hydrocarbons and metals. biphenyls, Major methodological breakthrough in recent years have enabled detailed genomic, metagenomic, proteomic, bioinformatics and other high throughput analysis of environmentally relevant microorganisms providing.

\section{Materials AND Method}

Sample Collection: Pesticide contaminated soils were collected from different areas in Tamil Nadu (Pazhani, Perampakkam, WCC College). Carbofuran 3CG was collected in local market in Thiruvallur. Tamil Nadu.

Isolation of Fungi from Contaminated Soil: To isolate pesticide degrading fungi from carbofuran contaminated soils. Media used for fungi was PDA or SDA with $1 \mathrm{~g}$ of carbofuran pesticide per $100 \mathrm{ml}$ (carbofuran was dissolved in methanol, acetone and acetonitrile.) and kept it for incubation at $37^{\circ} \mathrm{C}$ for 7 days. Degradation medium for fungi synthetic medium was used. Cappuccinoet al (2002).

Identification of Selected Isolated: To identification of the fungi was done using LPCB staining. Cappuccino, et al (2002).

Mass Cultivation of Fungi: Actively growing fungal culture was cultivated on SD broth used as medium. The flasks were incubated at $28^{\circ} \mathrm{C}$ in the shaker incubated at $100 \mathrm{rpm}$ for 3 weeks.Harold $\mathbf{~ J}$. B (2002). 
Preservation of Culture: Fungi in the pure culture were preserved on the slant and preserved at $8^{\circ} \mathrm{C}$ with proper labeling. Harold J. B (2002).

Effect of Concentration of Pesticides: $10 \mathrm{ml}$ of MM medium was added in four sets, each of six test tubes, autoclaved. Test tubes were inoculated with the isolated organisms and incubated at different concentration such as $0.5,1,1.5$ and 2 . Their absorbance was measured at $640 \mathrm{~nm}$ in colorimeter.

Thin Layer Chromatography: To determine degradation of fungi by TLC method. The solvent chloroform: ethyl acetate (3:1) was used. Nair,et al (2009). The Retention factor $\left(\mathrm{R}_{\mathrm{f}}\right)$ of the compound was calculated using the formula(Slaoui . et al2007)

$$
\mathrm{R}_{\mathrm{f}}=\frac{\text { Distance travelled by the compound }}{\text { Distance travelled by the solvent front }}
$$

High Performance Liquid Chromatography: A standard prepared by dissolving the standard pesticide in acetonoitrile to the final concentration. Carbofuran detected at $254 \mathrm{~nm}$ at a run time of 5 mins at a flow rate of $1 \mathrm{ml}$ per min using HPLC grade acetonitrile (70\%) and degassed water $(30 \%)$ as the mobile phase. Sample of $2 \mu \mathrm{l}$ is injected at an oven temperature of $270^{\circ} \mathrm{C}$. This has been done at a wavelength of $254 \mathrm{~nm}$ at a run time of 5 mins. (Slaoui .et al2007) (Seo. et al2007)

\section{RESULT AND DISCUSSION}

Isolation of Fungi from Pesticide Contaminated Soil: From the pesticide contaminated soil five different types fungi were isolated. They can able to grow and degraded in synthetic medium and PDA medium with carbofuran pesticide containing in that medium the resulted that growth is observed in 7days.Fig:1

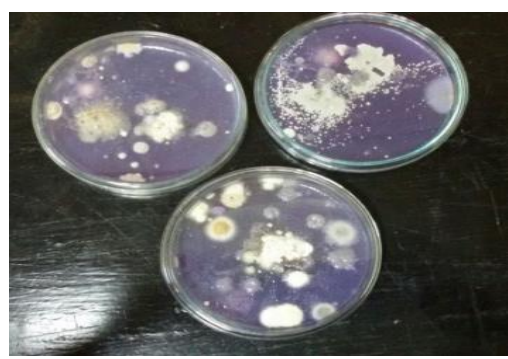

Fig1. Fungi isolated from the pesticide contaminated soil

Large Scale Cultivation: Mass cultivation of fungi isolates with carbofuran was done for further studiesFig:2.

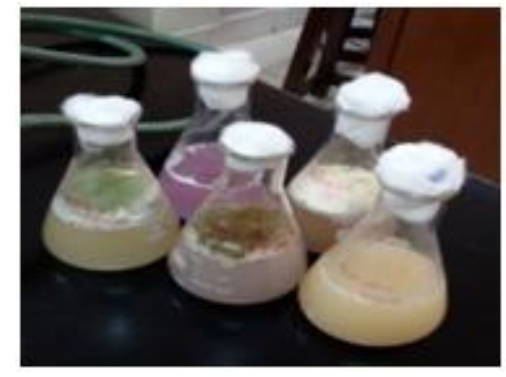

Fig2. Fungi large scale cultivation

Staining: The staining technique was used to know about the microorganisms fungi was isolated from pesticide contaminated soil.

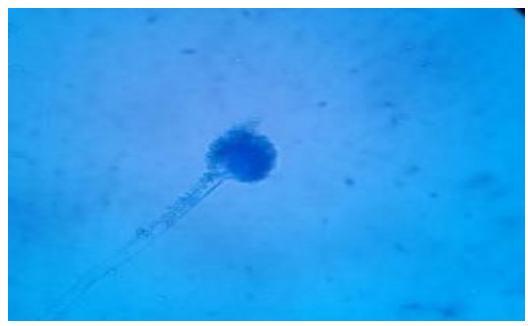

Fig3. Aspregillus spp 
Effect of Concentration of Pesticide for Fungi: The optimum concentration of pesticide for the growth of fungi was found to be concentration -1 and extreme temperatures concentration- 0.5 , concentration -1.5 and concentration -2 restricted the bacterial growth. At the rate of the best growth was found to be the highest $(\mathrm{OD}=0.53)$. The are observed for 3 days periodically.

TLC : The Retention factor $\left(\mathrm{R}_{\mathrm{f}}\right)$ of the compound was calculated using the formula

$$
\mathrm{R}_{\mathrm{f}}=\frac{\text { Distance travelled by the compound }}{\text { Distance travelled by the solvent front }}
$$

The analysis in TLC results showed that the retention time of Aspergillus spp is 0.10 and standard carbofuran is 0.70 . The analysis thin layer chromatography shows the presence of breakdown products. (Slaoui .et al2007)

HPLC: Carbofuran was biodegraded forming identified at the retention times for standard 5.863 minutes, and fungi retention times is 0.521 .Using independent enrichment step with carbofuran as the sole carbon and energy, fourteen carbofuran-degrading bacteria were isolated. The ability of the isolates to utilize carbofuran as sole carbon source was assessed using HPLC over a period of 120 days with the reduction in the concentration of carbofuran determined against carbofuran standard curve. The extent of degradation varied among the isolates. Growth of isolates was also monitored against change in concentration of carbofuran. (Seo. et al2007).(Slaoui .et al2007)

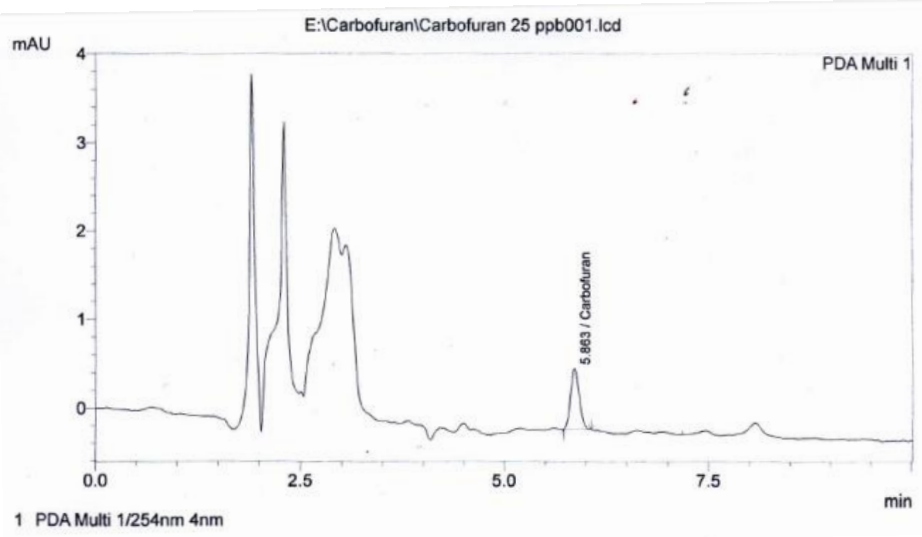

Fig4. HPLC STANDRAND FOR CARBOFURAN

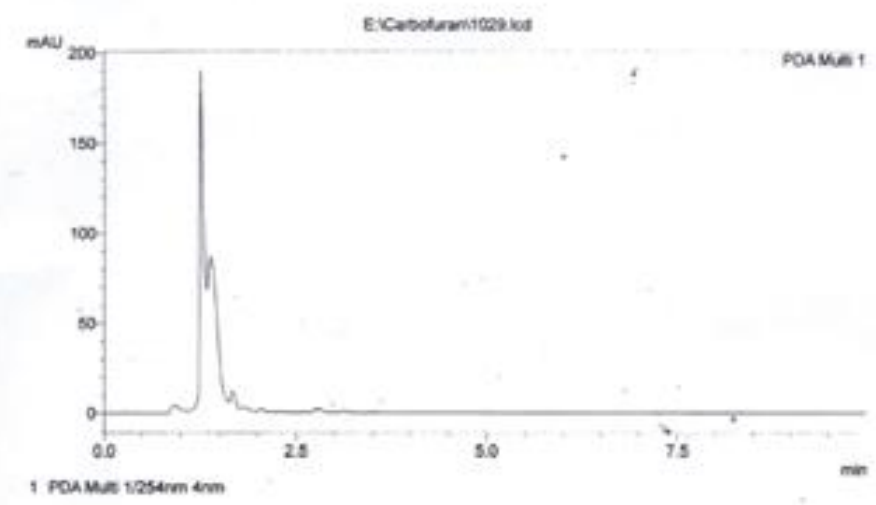

Fig5. HPLC FOR Aspergillus spp DEGRADING CARBOFURAN

\section{Conclusion}

This discussion suggests that the isolated organisms can flourish in the carbofuran pesticide using farms by utilizing them as their source of energy when others source are limited or unavailable. The isolation of carbofuran degrading fungi from hill soils and different areas in Tamil Nadu . From the study it has been confirmed that the organisms are capable of degrading the pesticide. In this analysis, used the several species of fungi, used the identified species called Aspergillusspp, Penicilliumsppand Trichotheriumspp. With help fungi, soil fertility can be increased and will remove the pesticide contamination. Using HPLC, the ability of degradation capability of fungi was monitored and it can able to degrade carbofuran pesticide. 


\section{REFERENCES}

Afify.A.E.M.MR., Seoud. M.A.A.E., Ibrahim. M.G, Kassem. W.B. (2013) stimulating of biodegradation of oxamyl pesticide by low dose gamma irradiated fungi. Plant pathology \&microbiology vol-4, ppl-5

Afzal.H., Shazad.S. and Nisa.S.Q.U.(2013). Morphological identification of Aspergillus species from the soil of larkana district (sindh, Pakistan).Asian J AgriBiol,vol-1(3)pp 105-11

Bailey, H.C., DiGiorgio, C., Kroll, K., Miller, J., Hinton, D.E., and Starrett, G. (1996). Development of Procedures for Identifying Pesticide Toxicity in Ambient Waters: Carbofuran, Diazinon, Chlorpyrifos. Environmental Toxicology Chemistry, 15(6):837-845

Cappuccino, J.G and Sherman, N (2002). Microbiology: a laboratory manual (6th ed) Pearson

Gibson, J. and Harwood, C.S. (2002). Metabolic diversity in aromatic compounds utilization by anaerobic microbes.Annual Review Microbiology, 56: 345-369

Griffiths, B.S., Ritz, K., Ebblewhite, N., Dobson, G., (1999). Soil microbial community structure: effects of substrate loading rates. Soil Biology and Biochemistry, 31:145- 153.

Hai.J.F,Modin.O,Yamamoto.K,Fukushi.K,Nakajima.F.and Nghiem.D.L.(2012) Pesticide removal by a mixed culture of bacteria and white rot fungi. Journal of the Taiwan institute of chemical engineers voOl.43 pp 459-462.

Hajslova, (1999). Environmental Contaminants in Food. Sheffield Academic Press, Chapter 7, 215

Hans, D. (1994). Topics in applied chemistry. Plenum press, Pittsburgh, PA. USA.

Harold J. B (2002). Microbiological applications: a laboratory manual in general microbiology. 8th edition. 1-478.

Hassan Shahgholi., (2014), Factors Controlling Defradation of Pesticides in the Soil Environment Review, TI Journals Agriculture Science Developments, Vol (3), No(8), pp.273-278

Heider, J and Fuchs, G. (1997).Anaerobic metabolism of aromatic compounds. European Journal of Biochemistry, 243:577-596.

Hindumathy.CK and Gayathri.V., (2013) Effect of Pesticide (Chlorpyrifos) on Soil Microbial Flora and Pesticide Degradatin by Strains Isolated from Contaminated Soil, Bioremediation \&Biodefradatin, Volume 4, Issue 2, 1-6

Mahony, M.M., Dobson, A.D. Barnes, J..D. and Singleton I. (2006). The use of ozone in the remediation of polycyclic aromatic hydrocarbon contaminated soil. Chemosphere, 63: 307-314.

Rakhee Nair, Priya.R.Iyer., (2009), Biodegradation-end of the Raod for Endosulfan, Environmental Science an Indian Journal, 4(2), (69-71)

Roger.P.A., Simpson.I., Oficial.R., Ardales.S. and Jimenez.R, (1994), Effects of Pesticides on soil and Water Microflora and Mesofauna in Wetland Ricefields: A summary of Current Knowledger and Extrapolation to temperate Environments, Australian Journal of experimental Agriculture, 34, 1057-68

Seo.J, Jeon.J, Kim.D.S.,Kang.S, Han.J., Hur.G.H..,(2007)Fungal biodegratation of carbofuran and carbofuran phenol by the fungus Mucor Ramannianus: identification of metabolites, water \& science technology, vol55nol-2pp163-167.

Slaoui.M., Ouhssine.M., Berny.E. and Elyachiouu.,(2007),Biodegradation of the Carbafuran by a Fungus Isolated fro Treated Soil, African Journal of Biotechnology, Vol.6(4), pp.419-423

Sriranjini.A.S., Michelle Samantha Fernandis, Savita Desai, (2012) Study and Identificatin of Potent Defrader of Chlorpyrifos Pesticide, International Journal of Research and Reviews in Pharmacy and Applied Science, 2(5) 847-858

Wootton.A.M, Kremer.J.R, Keaster.J.A.(1992).Effect of carbofuran and the corn rhizosphere on growth of soil microorganisms .Environmental contamination and toxicology.vol.50. pp49-56. 